\title{
Hand-written Numeral Recognition Based on Probabilistic Neural Network
}

\author{
Zheng Jun ${ }^{1, a}$ \\ ${ }^{1}$ Baotou Teachers'college Network Information Center, Inner Mongolia Baotou 014030, China \\ azhengjun_025@163.com
}

Keywords: probability neural network; hand-written numeral recognition; model recognition

\begin{abstract}
This paper analyzes favorable properties and realization principles of probabilistic neural network and elaborates the original image pre-processing and normalization processing of hand-written numeral, proper vector extraction, network model building, and other key techniques. Finally, this paper checks the model of hand-written numeral recognition based on probabilistic neural network, the results of which show that this model has high recognition accuracy and strong stability.
\end{abstract}

\section{Introduction}

We are living in a numeric society and deal with numerals everyday, such as student number, working number, bank card number, express number, and so on. It is rather inefficient to recognize in front of so much hand-written numeral information. Therefore, a kind of hand-written numeral recognition method with high efficiency and accuracy is necessary, which can be used in following situations. $\Phi$ Large-scale data statistics. In large-scale data statistics, abundant data needs to be recognized in short time with accuracy, such as industrial almanac, nationwide census, and other statistical activities. 2 Financial field. With the rapid development of economy, a large amount of financial statements, tax reports, checks, payment orders, and other hand-written numerals to be dealt with, are produced everyday. Efficient hand-written numeral recognition methods can save plenty of manpower and materials. Therefore, the study of hand-written numeral recognition has great realistic significance and broad application prospects.

Artificial neural network derives from a kind of mathematical model with nonlinear dynamic system which can realize information control and processing in cerebral neural network. Probabilistic neural network is a branch of artificial neural network, and classifies objects based on Bayes Minimum Risk Theory. This method has high accuracy, good instantaneity, and strong anti-jamming capacity. Therefore, this paper adopts probabilistic neural network to make studies on hand-written numerals.

\section{Overview on probabilistic neural network}

Probabilistic neural networks, PNN, is developed from RBF neural network by Doctor D.F.Specht in 1989. Its features are using linear learning algorithm to complete the work that can be done by nonlinear learning algorithm previously in the process of solving classification problems and keeping the high accuracy of nonlinear algorithm at the same time; the corresponding weight of this kind of network is the distribution of pattern samples, and network can meet the requirements of real-time processing in training without previous training. The main idea of probabilistic neural network is

Bayesian Decision Theory. Suppose binary classification problem $d(X)=\theta_{A} d(X)=\theta_{A}$, $X$ is p dimension sample vector $\mathrm{X}=\left(\mathrm{X}_{1}, \mathrm{X}_{2}, \ldots, \mathrm{X}_{\mathrm{p}}\right)^{\mathrm{T}}$, according to Bayesian Decision Theory, its classification model can be described as: 


$$
d(X)= \begin{cases}\theta_{A}, & h_{A} l_{A} f_{A}(x)>h_{B} l_{B} f_{B}(x) \\ \theta_{A}, & h_{A} l_{A} f_{A}(x)<h_{B} l_{B} f_{B}(x)\end{cases}
$$

$\mathrm{h}_{\mathrm{A}}$ and $\mathrm{h}_{\mathrm{B}}$ are prior probabilities of $\mathrm{X}={ }^{\theta_{\mathrm{A}}}$ and $\mathrm{X}={ }^{\theta_{\mathrm{B}}}$ respectively; ${ }_{\mathrm{A}}$ is the damage which is classified to ${ }^{\theta_{\mathrm{B}}}$ by mistake and $\mathrm{X}$ belongs to ${ }^{\theta_{\mathrm{A}}}$, and ${ }^{\mathrm{l}_{\mathrm{B}}}$ is the damage which is classified to ${ }^{\theta_{\mathrm{A}}}$ by mistake and $\mathrm{X}$ belongs to ${ }^{\theta_{\mathrm{B}}} ; \mathrm{f}_{\mathrm{A}}(\mathrm{x})$ and $\mathrm{f}_{\mathrm{B}}(\mathrm{x})$ are the probability density functions of $\theta_{\mathrm{A}}{ }_{\text {and }} \theta_{\mathrm{B}}$ respectively.

\section{Realization of hand-written numeral recognition based on probabilistic neural networks}

Data preparation:This study adopts the binary images of numbers from 0 to 9 as the training samples of probabilistic neural network. Each number from 0 to 9, 100 hand-written numeral images are collected with the resolution ratio of each image 64×64; there are 1000 training samples in all. Part of the training samples can be seen in Figure 1:

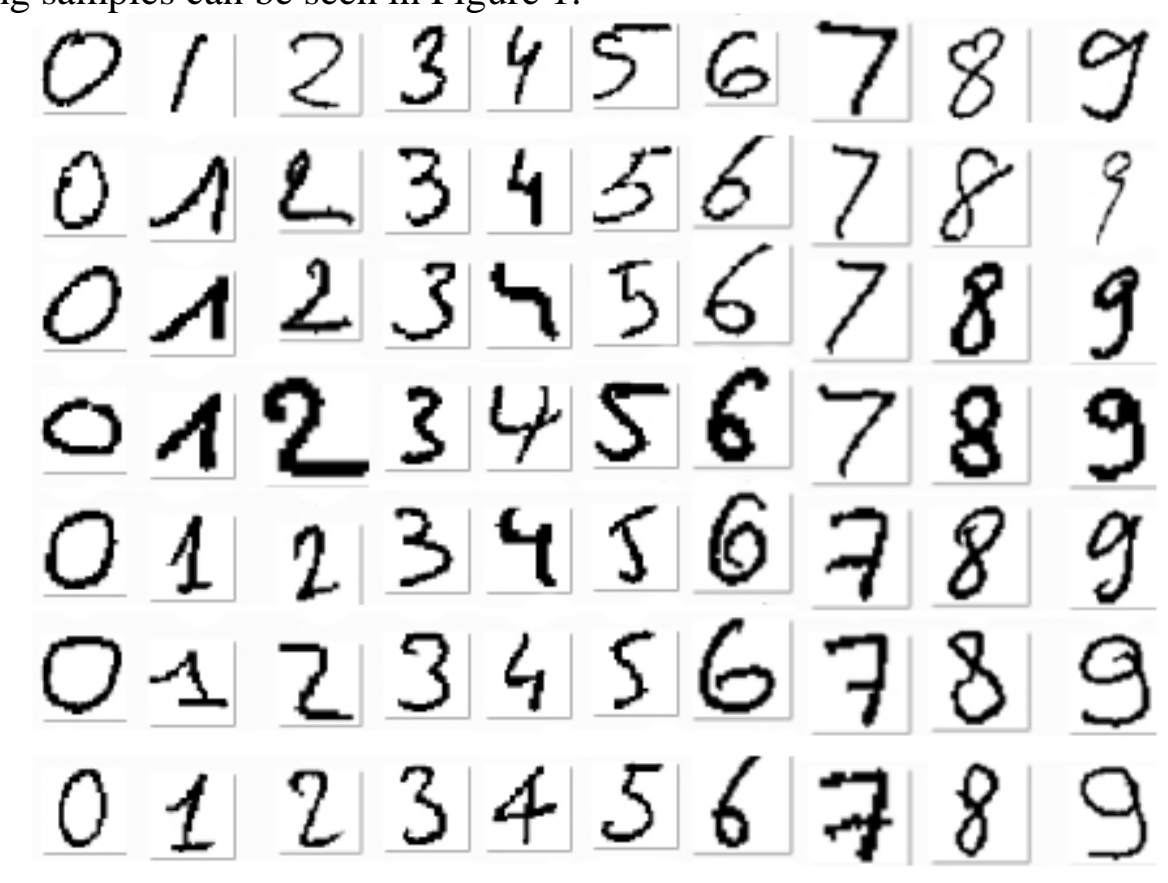

Figure 1 Part of training sample images

As the collected hand-written numeral images often contain various noises and stains; to avoid disturbance on recognition, de-noising processing is necessary to be done on original images. In this study, we adopts median filtering techniques to make de-noising processing on original images.

Numeral normalization:To decrease the influence of the shape and size of hand-written numerals on the numeral recognition, and to make sure that the proper value can be extracted more accurately later, normalization processing is needed to be done on data. Through normalization processing on data, we can make different numerals have the same size. The normalization algorithm this research

adopts is as following: suppose the top left coordinate of original image is ( $\mathrm{m}_{1}, \mathrm{n}_{1}$ ), the origin is $(\mu, \delta)$ and the height and width are $\mathrm{x}$ and $\mathrm{y}$ respectively; after normalization, the height and width are $\mathrm{X}$ and $\mathrm{Y}$ respectively and the top left coordinate remains the same. The pixel point $(\mathrm{m}, \mathrm{n})$ in the original image becomes $\left(\mathrm{m}^{I}, \mathrm{n}^{I}\right)$ in the new image after normalization: 


$$
\mathrm{m}^{I}=\mathrm{X} \times \frac{\left(\mathrm{m}-\mathrm{m}_{1}\right)}{\mathrm{x}}+\mu, \mathrm{n}^{I}=\mathrm{Y} \times \frac{\left(\mathrm{n}-\mathrm{n}_{1}\right)}{\mathrm{y}}+\delta
$$

In this research, after normalization, the height $\mathrm{X}$ and width $\mathrm{Y}$ of the image are both 64 . Through the steps above, we can get the hand-written numerals after pre-processing.

Feature extraction:If the image data after pre-processing are taken as the input of probabilistic neural network, on one hand, the data size in the input network becomes rather large, and on the other hand, the diversity of handwriting and certain interference in the images and pre-processing make a large amount of nonessential data go into the network, which makes the network too large and not strong enough. Therefore, we need to extract the proper vectors that can reflect hand-written numerical characteristics best and replace hand-written numerals with proper vectors as the input data of network. Thus, the quality of the extraction of proper vectors directly influence the classification effect of algorithm recognition. The selection method of proper vector in this research is as following: 1) Divide the character image into four equal parts in horizontal direction and two equal parts in vertical direction. Therefore, we get 8 equal areas; calculate number of pixel points with the pixel value of 255 in the 8 areas and get a 8-dimension feature, with the cutting method as Figure 2(a).

2) Draw two bisectrixes in horizontal and vertical directions respectively and calculate the number of points with pixel value of 255 in these four cutting lines; we can get a 4-dimension feature, as is shown in Figure 2(b).

3) Calculate the number of points with pixel value of 255 in the whole image, and we can get a 1-dimension feature, as is shown in Figure 2(c).
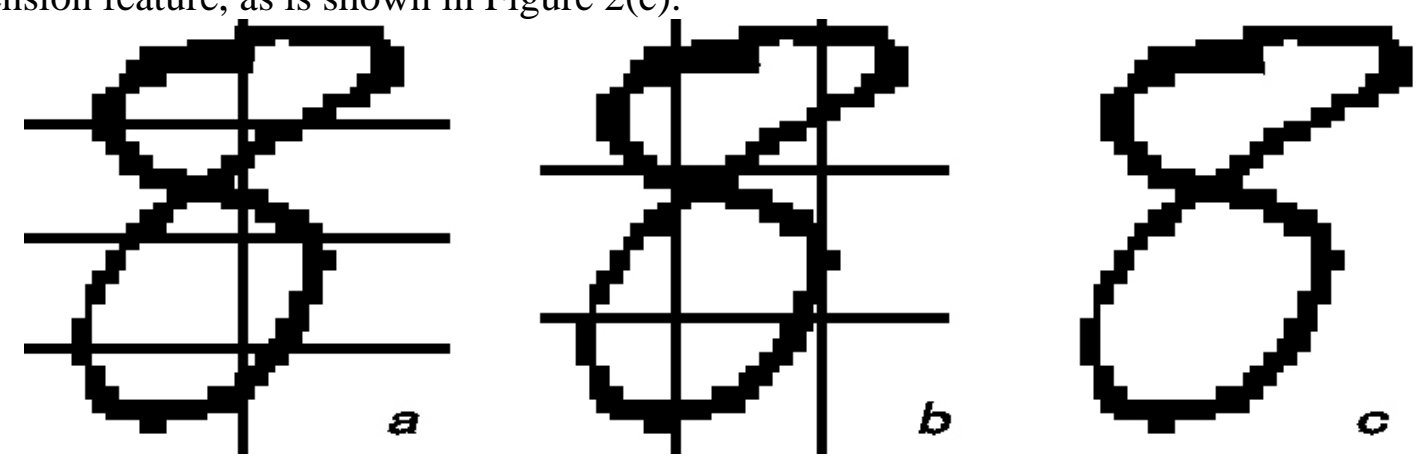

Figure 2 Diagram of feature extraction

Through step 1), 2) and 3), we can get 13-dimension proper vectors.

Modeling of probabilistic neural network:The network model of hand-written numeral recognition based on probabilistic neural network is shown in following figure:

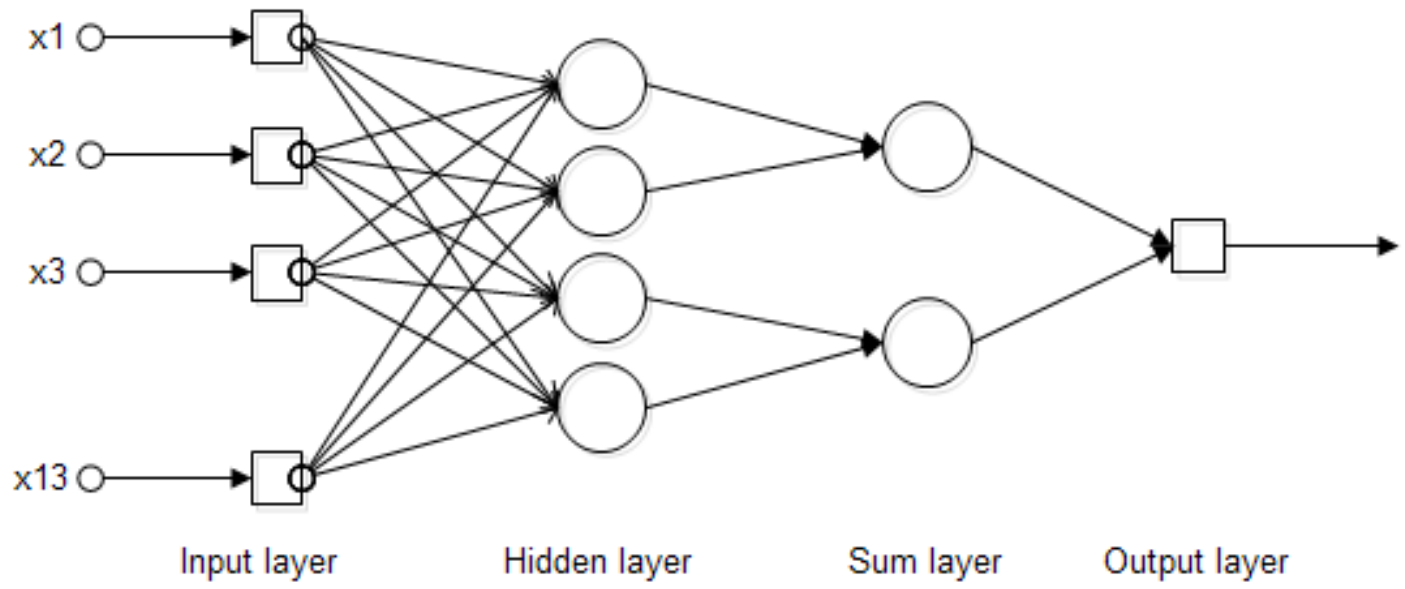

Figure 3 Diagram of network model structure

The model is composed of input layer, hidden layer, sum layer and output layer. Input layer is used to receive proper value vector of hand-written numerals, and it is composed of 13 neurons.

Hidden layer is used to receive the sample input in input layer, and one new neuron is added after receiving each sample input; as this study adopts 1000 training samples, the number of neurons in the 
hidden layer is 1000 . After the model training of hidden layer network, the vector $\mathrm{x}$ is input to hidden layer; the output model of neuron $\mathrm{j}$ in category $\mathrm{i}$ of hidden layer is as following:

$$
\Phi_{\mathrm{ij}}=\frac{1}{(2 \pi)^{\frac{1}{2}} \sigma^{\mathrm{d}}} \mathrm{e}^{\frac{\left(\mathrm{x}-\mathrm{x}_{\mathrm{ij}}\right)\left(\mathrm{x}-\mathrm{x}_{\mathrm{ij}}\right)^{\mathrm{T}}}{\sigma^{2}}}
$$

In $\mathrm{i}=1,2, \ldots, \mathrm{M}, \mathrm{M}$ is the category number of training samples, the value of $\mathrm{M}$ in this research is 10 ; $\mathrm{d}$ is the number of dimensions of sample space data; ${ }^{\mathrm{x}_{\mathrm{ij}}}$ is the center $\mathrm{j}$ of category $\mathrm{i} ;{ }^{\sigma}$ is smooth factor, whose value has important influence on the network performance.

Sum layer makes weighted average of output of the neurons in hidden layer that belong to the same category, and the number of neurons is the same as category $\mathrm{M}$, which means the number of neurons in sum layer is 10, with mathematical model as:

$$
v_{i}=\frac{\sum_{j=1}^{L} \Phi_{i j}}{L}
$$

The output of category $i$ is shown as $v_{i}$; $L$ stands for the number of neurons in category $i$.

Output layer is used to output recognition results of hand-written numerals, whose principle is to get the category with the largest value in sum layer as recognition result output, with the mathematical model as:

$$
\mathrm{y}=\operatorname{argmax}\left(\mathrm{v}_{\mathrm{i}}\right)
$$

\section{Experimental results and analysis}

Step one, we adopt training sample to make detection on the network model built above and the detection result is $100 \%$ right. Step two, we make another 50 hand-written numerals (as is shown in Figure 4) as test data and put in the network model to make detection on network model, with detection result as $100 \%$ right.

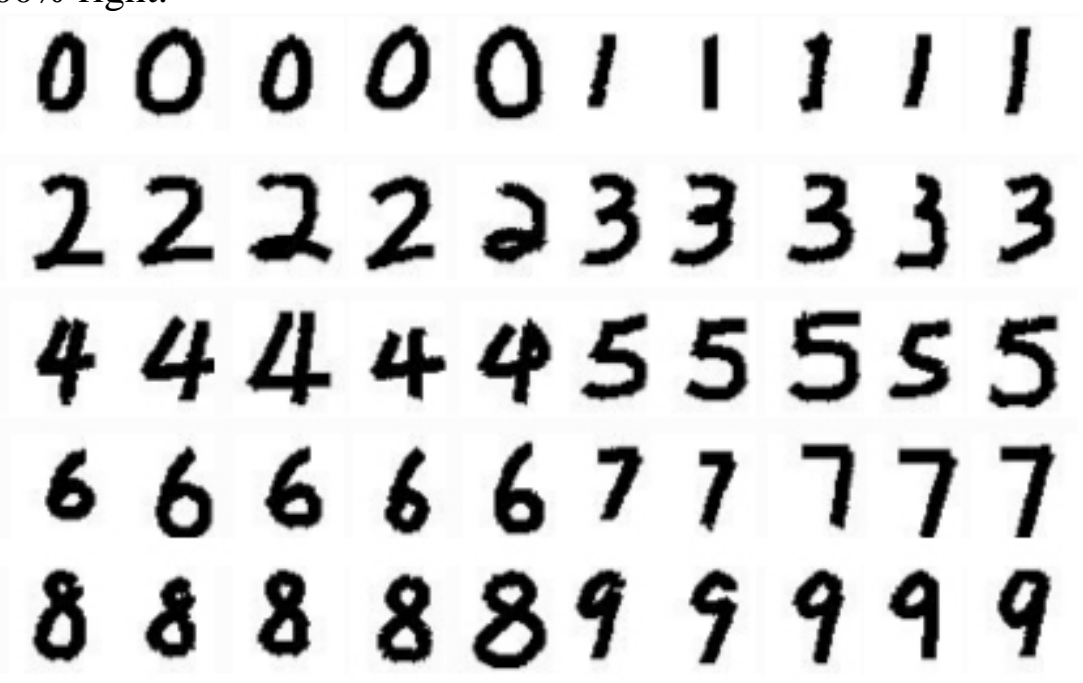

Figure 4 Example of test samples

Step 3, we add some interference to the 50 hand-written numerals that we make afterwards (as is shown in Figure 5) and make detection again, with detection result still as $100 \%$ right. 


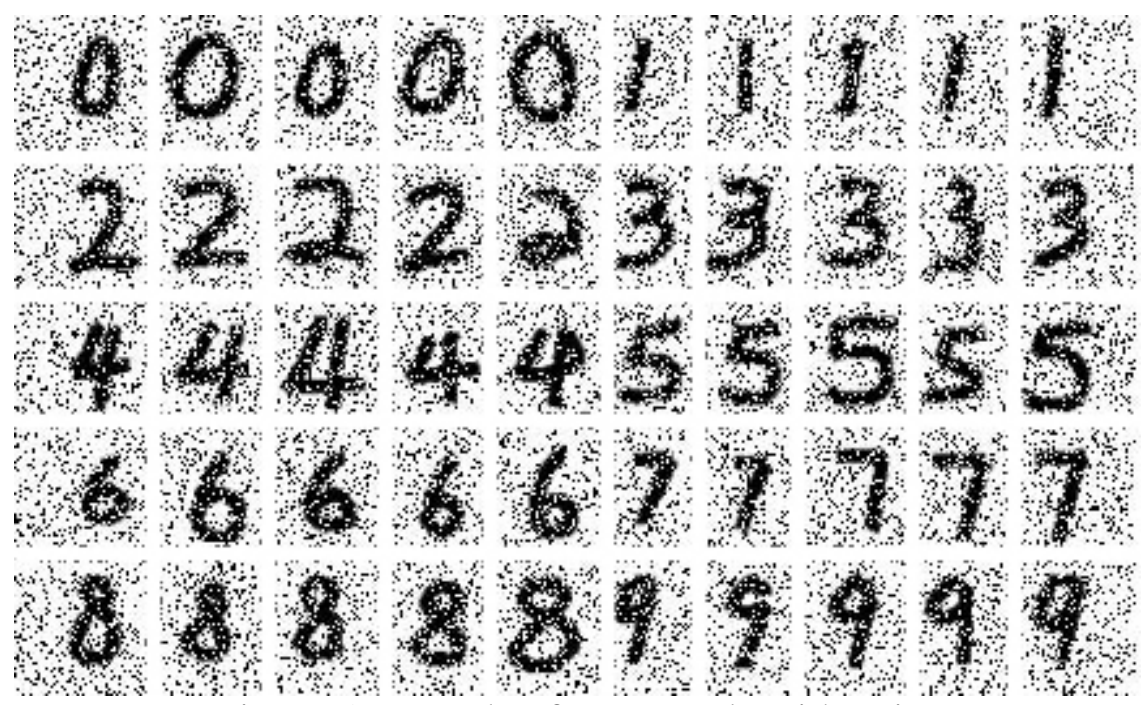

Figure 5 Example of test sample with noise

From the experiment above, it can be seen that hand-written numerals can be easily recognized by the hand-written numeral recognition algorithm based on probabilistic neural network and the anti-interference ability is strong.

\section{Reference}

[1] Ye Mingsheng, Wang Ailing, Deng Qiuxiang. Principle and application of handwritten numeral [M] Beijing: Tsinghua University press, 2008

[2] Han Liqun. Theory and application of artificial neural network [M]. Beijing: Chemical Industry Press, 2002

[3] Hagen. neural network design [M]. Beijing: Mechanical Industry Press, 2002:286-300

[4]Anthony Zaknich Introduction, to the modified probabilistic neural network for general sigal processing applications [J]. IEEE Trans on Neural Network, 1998,46 (7): 1989 - 1980

[5]Specht D F Probabilistic, neural networks[J] Neural, Networks, 1990, 3, 2, 109-118

[6]D Wellman, M.P. Bayesian Networks [J]. Communications of the ACM Heckerman, 38 (3). 1995 27-30

[7] Wang Shuangcheng, Zhou Yanjun, Wang Hui. Research on the classification of [J]. Northeast Normal University Journal natural science, 2003, 35, 6 (2): 21-27

[8] Jin Jian et al. A model of digital recognition system of artificial neural network based on feature. Journal of Nanjing Institute of meteorology, 2004, 27 (6): 829-835

[9]V.K. Govindan A.P, . Shivaprasad, Recognition-A Review[J] Pattern, Recognition. 1990,23 Character (7): 671-683

[10] Qin Zhen Xu, Yang Lu Xi. Handwritten digits recognition method [J]. Journal of electronics and information technology based on improved hybrid learning model, 2010, 26 (4)433-438

[11] Dong Hui. Feature extraction and feature selection in handwritten numeral recognition [D]. Beijing University of Posts and Telecommunications, 2007 